H.-G Schöpf

Von Kirchhoff bis Planck 


\section{REIHE WISSENSCHAFT}

Die REIHE WISSENSCHAFT ist die wissenschaftliche Handbibliothek des Naturwissenschaftlers und Ingenieurs und des Studenten der mathematischen, naturwissenschaftlichen und technischen Fächer. Sie informiert in zusammenfassenden Darstellungen über den aktuellen Forschungsstand in den exakten Wissenschaften und erschließt dem Spezialisten den Zugang zu den Nachbardisziplinen. 
Hans-Georg Schöpf

\section{Von Kirchhoff bis Planck}

Theorie der Wärmestrahlung in historisch-kritischer Darstellung

Mit 9 Abbildungen

V

Springer Fachmedien Wiesbaden GmbH 
Herausgeber dieses Bandes:

Prof. Dr. Hans-Georg Schöpf

Sektion Physik der Technischen Universitat Dresden

CIP-Kurztitelaufnahme der Deutschen Bibliothek

Schöpf, Hans-Georg

Von Kirchhoff bis Planck: Theorie d. Wärmestrahlung in histor.-krit. Darst. - 1. Aufl. Braunschweig: Vieweg, 1978.

(Reihe Wissenschaft)

\section{8}

Akademie-Verlag Berlin 1978

Alle Rechte vorbehalten

(c) Springer Fachmedien Wiesbaden 1978

Ursprünglich erschienen bei Friedr. Vieweg \& Sohn Verlagsgesellschaft $\mathrm{mbH}$, Braunschweig 1978

Softcover reprint of the hardcover 1st edition 1978

Lizenzausgabe fur

Friedr. Vieweg \& Sohn Verlagsgesellschaft mbH, Braunschweig, mit Genehmigung des Akademie-Verlages, DDR - Berlin Herstellung: VEB Druckhaus „Maxim Gorki", 74. Altenburg

ISBN 978-3-528-06840-0

ISBN 978-3-663-13885-3 (eBook)

DOI 10.1007/978-3-663-13885-3 


\section{Vorwort}

Im Lehrbetrieb der theoretischen Physik (und nicht nur dieser) werden augenscheinlich Behauptungen mathematisch-physikalischen oder ähnlichen Inhalts und eingeflochtene historische Bemerkungen mit durchaus unterschiedlicher Sorgfalt behandelt. Im ersten Fall wird der Dozent, sofern er den Anspruch erhebt, seriös zu sein, in der Regel nichts vortragen, von dessen Richtigkeit er sich nicht vorher persönlich überzeugt hätte, obwohl er dabei im allgemeinen die Darlegungen der Lehrbücher bestätigt findet. Historische Bemerkungen hingegen werden nur allzu oft kritiklos von den Lehrern übernommen und an die Schüler weitergetragen, obwohl ein Blick in die Quellen zeigen würde, daß es sich in der Regel um Geschichtslegenden handelt. Als ich aus Anlaß des 75 . Jubiläums der PLanckschen Quantenhypothese einen Gedenkvortrag konzipierte, wurde mir das deutlich bewußt, und ich fühlte mich bewogen, das Quellenstudium auf die Vorgeschichte auszudehnen. Sie beinhaltet die Entwicklung der Wärmestrahlungstheorie, die im Zeitraum von 1860 bis 1900 erfolgte und durch die Namen Kirchhoff, Boltzmann, W. Wien, Rayleigh und PLanck gekennzeichnet ist. Aus der Beschäftigung mit den Werken dieser großen Physiker hat sich dann im Laufe der Zeit das vorliegende Büchlein entwickelt.

Seine wissenschaftshistorische Prätention ist bescheiden. Wir hoffen, wenigstens die gröbsten der gängigen Irrtümer über den historischen Sachverhalt richtiggestellt zu haben. Jedoch war kein Platz, um auf dem allgemeinen gesellschaftlichen und physikgeschichtlichen Hintergrund das persönliche Schicksal, den persönlichen 
Stil der erwähnten Forscher abzuheben und ihren Beitrag zum wissenschaftlichen Fortschritt allseitig zu würdigen.

Aber ihren hier nachgedruckten Texten stellen wir auch nicht einfach eine ,,moderne Darstellung“ gegenüber, als ob es außer Diskussion stünde, daß eine ,,moderne Darstellung" auch in jeder Hinsicht die beste und verständlichste sei. Wir sind im Gegenteil den Gedanken der Originalarbeiten nachgegangen, und dabei entstand gewissermaßen von selbst ein Grundriß der Wärmestrahlungstheorie in historischer Darstellung. Nichts könnte besser belegen, daß aller Anlaß besteht, die in ihrer Ursprünglichkeit lebendigen, ebenso tiefsinnigen wie elementaren Ideen und Methoden der großen Meister der Physik dem gegenwärtigen akademischen Lehrbetrieb nutzbar zu machen.

In diesem Sinne würden wir es sehr begrüßen, wenn auch Studenten dieses Büchlein zur Hand nähmen. Dessen eingedenk haben wir versucht, den Inhalt so zu gestalten, daß er möglichst ohne spezielle Vorkenntnisse verständlich ist. Das war auch ein Grund dafür, das kurze Kapitel über die BoLTzmannsche Gasstatistik aufzunehmen. Ein anderer Grund ist natürlich ihr Einfluß auf die Entwicklung der Strahlungstheorie.

Selbstverständlich kann auch in der Physik die Rezeption des klassischen Erbes nicht kritiklos erfolgen. Lassen die Klassiker in manchen Punkten durchaus mehr Sorgfalt als die heutige Lehrbuchliteratur walten, so gibt es andererseits Passagen, die uns in Hinblick auf die Diktion oder die mathematische Technik oder die logische Stringenz nicht zu befriedigen vermöchten. Wie wir in solchen Fällen verfahren sind, mag der Leser durch Vergleich unserer Darstellung mit den originalen Texten ersehen und dabei zugleich entscheiden, ob wir im Geist der Autoren gehandelt haben. In einem Fall, und zwar bei den Veröffentlichungen Plancks vor dem Herbst des Jahres 1900, haben wir uns auf den Nachdruck eines kurzen Auszugs beschränkt. Dafür sprach der große 
Umfang dieser Arbeiten, aber auch die Tatsache, daß sie ja nicht zum Ziel führten. Wir hielten es jedoch für angemessen, ihren Inhalt ausführlich zu referieren. Im historischen Rückblick nämlich erweist es sich nicht selten, daß ein großartiger Irrtum den Fortschritt der Wissenschaft mehr stimuliert hat als manche kleine Wahrheit.

In nicht wenigen Fällen bringt erst das Licht späterer Kenntnisse Wahrheiten zum Vorschein, die in den originalen Ansätzen impliziert sind, ohne daß sie schon gesehen werden konnten. Es ist eine legitime Aufgabe historisch-kritischer Analyse, solche Wahrheiten zu explizieren, wenn dabei auch Anachronismen in Kauf zu nehmen sind. Von dieser Art ist das 6. Kapitel dieses Büchleins, das die Interpretation der WIEnschen Strahlungstheorie als die eines klassischen Lichtteilchengases enthält. Man kann nicht über Wärmestrahlung schreiben, ohne den korpuskularen Aspekt des Lichts zu berücksichtigen. In engem Zusammenhang damit steht die Schwankungsproblematik. Wir haben sie im 7. Kapitel behandelt, das auch den gesteckten engeren historischen Rahmen überschreitet.

Die von den Autoren der reproduzierten Texte gewählten Bezeichnungen physikalischer Größen konnten selbstverständlich nicht angetastet werden. Zur Erleichterung für den Leser haben wir auf S. 195 die wichtigsten Symbole den von uns im ersten Teil benutzten gegenübergestellt. Wir hielten es für berechtigt, diese in einer historischen Darstellung an der über Jahrzehnte bewährten Planckschen Nomenklatur zu orientieren, wenn auch Identität weder möglich noch erstrebenswert war.

Die Orthographie der Originalarbeiten wurde dagegen durchweg der heute üblichen angepaßt. Das hat meine Frau bei der von ihr besorgten Herstellung des Manuskripts bewerkstelligt. Sie hat auch die benutzte Originalliteratur herangeschafft. Für ihren wesentlichen Beitrag zur Fertigstellung dieses Büchleins möchte ich ihr sehr herzlich danken. Dank gebührt ferner Herrn Professor H.-J. 
Trener, Potsdam-Babelsberg, der mich zu dieser Arbeit ermutigt hat und an ihrem Fortgang lebhaftes Interesse zeigte. Schließlich sei dem Akademie-Verlag für seine verständnisvolle Zusammenarbeit aufrichtig gedankt.

Dresden, 30. Oktober 1976

\author{
H.-G. SchöPF
}




\section{Inhaltsverzeichnis}

\section{Teil 1}

Kapitel 1. Gustav Kirchhoff und das Emissions-Absorptions-Gesetz . . . . . . . . . . . . 11

Kapitel 2. Ludwig Boltzmann und das $T^{4}$-Gesetz . . . . 29

Kapitel 3. WILly WIEN und das Verschiebungsgesetz . . . 36

Kapitel 4. Willy Wien, Max Planck und das Energieverteilungsgesetz . . . . . . . . . 45

Kapitel 5. Ludwig Boltzmann und die elementare Gasstatistik . . . . . . . . . . . . . . . . . 68

Kapitel 6. Albert Ernstern und das klassische Lichtteilchengas . . . . . . . . . . 76

Kapitel 7. Lord RAYLEIGf und die Eigenschwingungen des Hohlraums . . . . . . . . . . . . . . . . 85

Kapitel 8. Max Planck und das endgültige Strahlungsgesetz . . . . . . . . . . . . . . . 99

Kapitel 9. Max Planck und die Quantisierung des harmonischen Oszillators . . . . . . . . . 105

Teil 2

1. Über das Verhalltnis zwischen dem Emissionsvermögen und dem Absorptionsvermögen der Körper für Wärme und Licht

G. KIRCHHOFF

2. Ableitung des Stefanschen Gesetzes, betreffend die Abhängigkeit der Wärmestrahlung von der Temperatur aus der elektromagnetischen Lichttheorie . . . . . . 152 L. BoltzManN

3. Eine neue Beziehung der Strahlung schwarzer Körper zum zweiten Hauptsatz der Wärmetheorie . . . . . . 156 W. WIEN

4. Über irreversible Strahlungsvorgänge . . . . . . . 166 M. Planck 
5. Bemerkungen über das Gesetz der vollständigen Strahlung . . . . . . . . . . . . . . . 172 Lord RAYLEIGH

6. Über eine Verbesserung der Wrenschen Spektralgleichung . . . . . . . . . . . . . . . . 175 M. Planck

7. Über das Gesetz der Energieverteilung im Normalspektrum . . . . . . . . . . . . . . . . . 178 M. Planck

8. Über die Elementarquanta der Materie und der Elektrizität . . . . . . . . . . . . . . . . . . . . . . 191 M. Planck

Zur Bezeichnungsweise . . . . . . . . . . . . . . . . 195 Sachverzeichnis . . . . . . . . . . . . . . . . 197 